\title{
Microscopic description of d-wave superconductivity by Van Hove nesting in the Hubbard model
}

\author{
J. González \\ Instituto de Estructura de la Materia. Consejo Superior de Investigaciones Científicas. Serrano 123, 28006 Madrid. Spain.
} (October 27, 2018)

\begin{abstract}
We devise a computational approach to the Hubbard model that captures the strong coupling dynamics arising when the Fermi level is at a Van Hove singularity in the density of states. We rely on an approximate degeneracy among the many-body states accounting for the main instabilities of the system (antiferromagnetism, $d$-wave superconductivity). The Fermi line turns out to be deformed in a manner consistent with the pinning of the Fermi level to the Van Hove singularity. For a doping rate $\delta \sim 0.2$, the ground state is characterized by $d$-wave symmetry, quasiparticles gapped only at the saddle-points of the band, and a large peak at zero momentum in the $d$-wave pairing correlations.
\end{abstract}

74.20.Mn,71.27.+a,71.10.Fd

Nowadays it is widely accepted that the single-band Hubbard model is an appropriate starting point to describe the electron correlations in the copper-oxide materials [1]. In spite of the efforts to show that the model has a phase of $d$-wave superconductivity, however, there is no conclusive proof at present of its existence. There have been different theoretical approaches to understand the symmetry of the order parameter, among which we may quote the proposals based on the effect of antiferromagnetic fluctuations [2] and the Kohn-Luttinger mechanism due to Van Hove nesting of the Fermi surface [3, 1 . Numerical methods have also provided some evidence of enhanced pairing correlations in the 2D Hubbard model [5,6]. Recent progress has been achieved by the use of dynamical mean-field approximations [7] and improved quantum Monte Carlo methods [8,9].

We focus our discussion on the proposal of superconducting pairing by the influence of a Van Hove singularity near the Fermi level [10]. Recently, some understanding of the problem has been attained by the use of refined renormalization group methods for interacting electrons in two dimensions 11],12]. This approach suffers, though, from the same shortcoming of any attempt to deal with the origin of high- $T_{c}$ superconductivity. The system is likely to develop strong antiferromagnetic or superconducting correlations but, as long as the effective interaction grows large as more high-energy electron modes are integrated out, it is not possible to discern rigorously the ground state of the model. A related problem concerns the fact that the superconducting correlations are enhanced like $\log ^{2} \varepsilon$, when the electron modes are integrated out down to energy $\varepsilon$ near the Fermi surface. This reflects in another fashion that the model does not lead to a conventional scale-invariant theory at arbitrarily low energies - unless the Fermi energy is promoted to a dynamical quantity susceptible itself of renormalization [13,12.

In this paper we address the characterization of the ground state of the Hubbard model, when the Fermi level

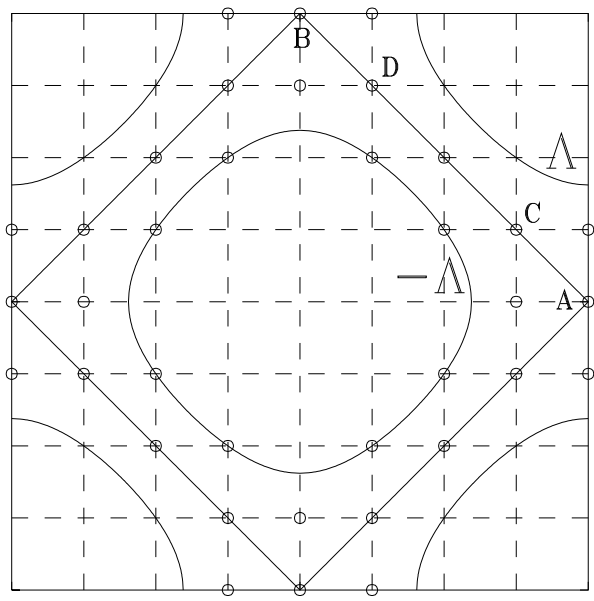

FIG. 1. Points of the $8 \times 8$ lattice in momentum space.

is at a Van Hove singularity in the density of states. For this purpose we will formally make use of the scaling properties of the model [13], which ensure that upon integration of the high-energy modes the electron quasiparticles remain in correspondence with the original oneparticle states, up to the energy in which the effective interaction starts to grow large. To elucidate the behavior of the system at lower energies we undertake a numerical diagonalization of the Hubbard hamiltonian, in the Hilbert space truncated to include the states left after integration of the high-energy modes.

We implement the above strategy in the Hubbard model on a $8 \times 8$ lattice, which has the set of one-particle states in momentum space represented in Fig. 11. In order to study the effects of the enhanced scattering at the saddle-points, we start from a Fermi sea of occupied states below the shell $\varepsilon=0$, whose particle content corresponds to a doping rate $\delta \approx 0.22$. We suppose that at a previous renormalization group stage the high-energy electron modes have been integrated out down to the region between energies $-\Lambda$ and $\Lambda$ about the Fermi level shown in Fig. 11. The modes integrated out have approx- 
imately isotropic patterns about the top and the bottom of the band, and we may safely assume that their effect is encoded in a soft renormalization of the effective coupling for the low-energy modes, that we call $U_{\text {eff }}$. Furthermore, we will deal with a situation in which $U_{\text {eff }}$ is in the weakcoupling regime, so that the low-energy physics can be described by building the many-body Hilbert space out of the one-particle states within $-\Lambda$ and $\Lambda$. One has to bear in mind, however, that the interaction of the states at the Fermi level shell $\varepsilon=0$ is always strong and essentially nonperturbative. In fact, we know from theoretical arguments that the main physical features have to come from the enhanced scattering with momentum transfer $(\pi, \pi)$. This effect is properly captured in our construction, given the high degeneracy of states at the $\varepsilon=0$ shell.

We proceed then to the numerical diagonalization in the many-body space of states of the low-energy theory, for different contents of particles at the Fermi level shell $\varepsilon=0$. We keep in all cases a Fermi sea of 24 particles filling the levels below $\varepsilon=0$. The instances with an even number of particles at the Fermi level are particularly interesting, since these are situations that allow to test the tendency to pairing in the model. Actually, there is a correspondence between the states with broken symmetry in a macroscopic system and the sectors labelled by different quantum numbers in the finite cluster [14]. The antiferromagnetic phase is characterized by a ground state in the sector A of spin singlets with total momentum $\mathbf{P}=(\pi, \pi)$ and all the quantum numbers equal to 1 for the rest of discrete symmetries of the lattice. The $d$-wave superconducting phase may be characterized by a ground state in the sector $\mathrm{D}$ of states with total momentum $\mathbf{P}=(0,0)$ and odd parity under the exchange of the two axes in momentum space (the rest of quantum numbers remaining equal to 1). A ground state belonging to the sector $\mathrm{P}$ of spin singlets with total momentum $\mathbf{P}=(0,0)$ and even parity for the rest of the symmetry operations implies a phase without symmetry breaking at the macroscopic level.

An important observation is that, for any even number of particles, the lowest-energy states found in the A, D and $\mathrm{P}$ sectors by considering the scattering of the oneparticle states in the $\varepsilon=0$ shell (and no particle-hole excitations from the Fermi sea) are always degenerate. Let us call $\mathbf{p}_{A}=(\pi, 0), \mathbf{p}_{B}=(0, \pi), \mathbf{p}_{C}=(3 \pi / 4, \pi / 4)$, $\mathbf{p}_{D}=(\pi / 4,3 \pi / 4)$. For two particles at the Fermi level, for instance, the following states have the same energy

$$
\begin{aligned}
|A\rangle & =\frac{1}{\sqrt{2}}\left(c_{\uparrow}^{+}\left(\mathbf{p}_{A}\right) c_{\downarrow}^{+}\left(\mathbf{p}_{B}\right)-c_{\downarrow}^{+}\left(\mathbf{p}_{A}\right) c_{\uparrow}^{+}\left(\mathbf{p}_{B}\right)\right)|F S\rangle \\
& -\frac{1}{2 \sqrt{8}} \sum_{\mathcal{R}}\left(c_{\uparrow}^{+}\left(\mathbf{p}_{C}\right) c_{\downarrow}^{+}\left(\mathbf{p}_{D}\right)-c_{\downarrow}^{+}\left(\mathbf{p}_{C}\right) c_{\uparrow}^{+}\left(\mathbf{p}_{D}\right)\right)|F S\rangle \\
|D\rangle & =\frac{1}{\sqrt{2}}\left(c_{\uparrow}^{+}\left(\mathbf{p}_{A}\right) c_{\downarrow}^{+}\left(\mathbf{p}_{A}\right)-c_{\uparrow}^{+}\left(\mathbf{p}_{B}\right) c_{\downarrow}^{+}\left(\mathbf{p}_{B}\right)\right)|F S\rangle
\end{aligned}
$$

$$
\begin{aligned}
\left|D^{\prime}\right\rangle & =\frac{1}{\sqrt{8}} \sum_{\mathcal{R}}\left(c_{\uparrow}^{+}\left(\mathbf{p}_{C}\right) c_{\downarrow}^{+}\left(-\mathbf{p}_{C}\right)-c_{\uparrow}^{+}\left(\mathbf{p}_{D}\right) c_{\downarrow}^{+}\left(-\mathbf{p}_{D}\right)\right)|F S\rangle \\
|P\rangle & =\frac{1}{\sqrt{2}}\left(c_{\uparrow}^{+}\left(\mathbf{p}_{A}\right) c_{\downarrow}^{+}\left(\mathbf{p}_{A}\right)+c_{\uparrow}^{+}\left(\mathbf{p}_{B}\right) c_{\downarrow}^{+}\left(\mathbf{p}_{B}\right)\right)|F S\rangle \\
& -\frac{1}{2 \sqrt{8}} \sum_{\mathcal{R}}\left(c_{\uparrow}^{+}\left(\mathbf{p}_{C}\right) c_{\downarrow}^{+}\left(-\mathbf{p}_{C}\right)+c_{\uparrow}^{+}\left(\mathbf{p}_{D}\right) c_{\downarrow}^{+}\left(-\mathbf{p}_{D}\right)\right)|F S\rangle
\end{aligned}
$$

where $\mathcal{R}$ stands for all possible reflections about the axes and $|F S\rangle$ represents the Fermi sea below the $\varepsilon=0$ shell. It can be checked that this degeneracy among the lowestenergy states in the three different sectors holds for any even number of particles in the Fermi level $\varepsilon=0$, when particle-hole excitations from the Fermi sea are frozen out.

Thus, in order to find to which sector belongs the ground state of the system, it suffices to make perturbations around the zeroth-order degenerate states. Wigner theorem establishes that no generic level crossings are found when just one parameter is varied in a quantum system 15. In our problem, we may switch adiabatically by means of an energy cutoff the number of particle-hole processes that are allowed in the space of states, in order to resolve the above degeneracy. The theorem guarantees that the ground state obtained in this evolution has to be adiabatically connected to the ground state of the full many-body space. In practice, it is enough to perturb the many-body states with up to two particle-hole excitations from the Fermi sea comprising 24 particles below $\varepsilon=0$. At this level, we may consider our computational method as accurate as the coupled-cluster-double approximation, in which the relative errors estimated for the ground state energy of the Hubbard model are as small as $\sim 10^{-4}$, for a $8 \times 8$ lattice and $U=t$ 16. As long as we are going to obtain our main results from the energy balance between different states in the model, we may expect them to be affected by smaller relative errors at that value of the interaction.

We have computed the lowest-energy states in the sectors with different quantum numbers, within the above approximation to the space of states, for a number $N$ of particles in the $\varepsilon=0$ shell ranging from zero to 4 . These values are about a doping rate $\delta \sim 0.2$. Otherwise, the case $N=4$ already sets the computational limit in the number of particles that can be afforded with our Fermi sea. The ground state with all the closed shells at $N=0$ is a spin singlet with momentum $\mathbf{P}=(0,0)$ and even parity for the rest of discrete symmetries. Its energy for $U_{\text {eff }}=t$ turns out to be $\approx-25.1713 t$. The competition between the lowest energies in the different sectors for $N=2$ and $N=4$ is illustrated in Table I1, for $U_{\text {eff }}=t$ and $2 t$. We have found that the ground state is always in the $\mathrm{D}$ sector of odd parity under the exchange of $p_{x}$ and $p_{y}$. This conclusion for $N=2$ can be considered a confirmation of the significance of similar results obtained by the full numerical diagonalization of the Hubbard model 
in a $4 \times 4$ cluster [17]. In the present approach, the Fermi level at $\varepsilon=0$ admits a larger number of particles, and it allows us to check the trend towards $d$-wave symmetry by filling the shell.

\begin{tabular}{|l|l|r|r|r}
\hline \hline & & $\mathbf{P}=(0,0)$ & $\mathbf{P}=(0,0)$ & $\mathbf{P}=(\pi, \pi)$ \\
& & $s$-wave & $d$-wave & \\
\hline$N=2$ & $U=t$ & -24.7985 & -24.7999 & -24.7985 \\
& $U=2 t$ & -22.4508 & -22.4540 & -22.4507 \\
\hline$N=4$ & $U=t$ & -24.4270 & -24.4273 & -24.4253 \\
& $U=2 t$ & -21.7124 & -21.7159 & -21.7063 \\
\hline \hline
\end{tabular}

TABLE I. Lowest energies of states with different quantum numbers, for particle content $N=2$ and $N=4$ in the $\varepsilon=0$ shell.

To get insight about the nature of the ground states at $N=2$ and $N=4$, we have looked at the quasiparticle excitations supported by them. These correspond in our cluster to states with an odd number of particles $(N=$ 1 and $N=3$ ). States with $N=1$ can be considered quasiparticle excitations over the fully symmetric state with $N=0$, and their dispersion may be obtained by looking at different momenta above the Fermi sea. We have plotted in Fig. 2 the lowest energies found for $U_{\text {eff }}=$ $t$ among the many-body states with different momenta on the shell $\varepsilon=0$ (including again up to two particle-hole excitations from the Fermi sea).

The Fermi energy for $N=1$ can be estimated by taking the mean value between the ground state energies at $N=0$ and $N=2$, which turns out to be $\approx-24.9856 t$ for $U_{\text {eff }}=t$. The lowest energy found at $\mathbf{P}=(\pi, 0)$ is slightly above this value, by an amount of order $10^{-4} t$. The quasiparticle excitations at $\mathbf{P}=\left(\frac{3 \pi}{4}, \frac{\pi}{4}\right)$ and $\mathbf{P}=\left(\frac{\pi}{2}, \frac{\pi}{2}\right)$ are found at higher energies. Results pointing in the same direction have been obtained in the diagonalization of small clusters [18]. We find a clear indication that the Fermi line is deformed by the interaction, in such a way that it is shifted inwards at the momenta $\left(\frac{3 \pi}{4}, \frac{\pi}{4}\right)$ and $\left(\frac{\pi}{2}, \frac{\pi}{2}\right)[19]$. This deformation is in agreement with the effect of pinning of the Fermi energy near a Van Hove singularity that has been discussed on theoretical grounds 20,3. According to this effect, the particles take advantage of the strong screening of the interaction at the singularity to place themselves preferently near the saddle points of the dispersion relation, as observed in our computation. A similar phenomenon has been advocated recently in Ref. 21.

It is also of great interest the study of the lowest-energy states for $N=3$ particles on the $\varepsilon=0$ shell. These may be considered as the quasiparticle excitations of the ground states for $N=2$ and $N=4$, and they are essential to characterize the pairing of particles at even filling levels. The energies for the momenta on the $\varepsilon=0$ shell, obtained in the same approximation as before, are plotted in Fig. 2 for $U_{\text {eff }}=t$.

The position of the Fermi energy at $N=3$ can be

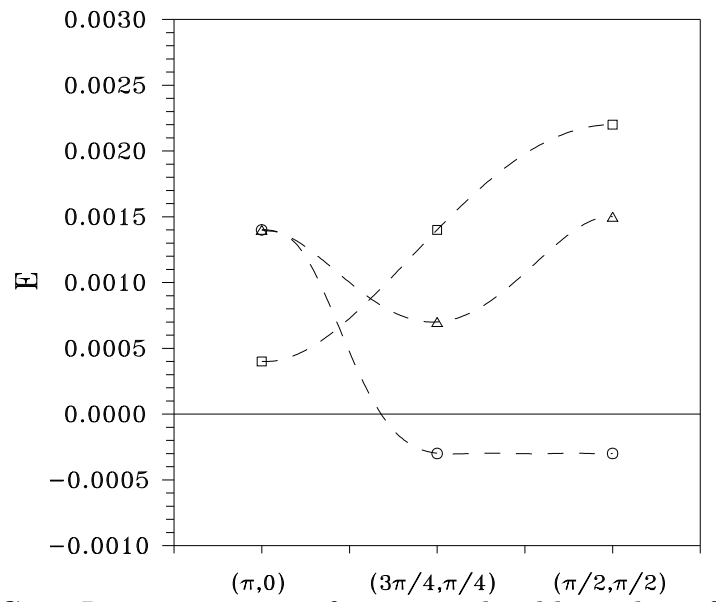

FIG. 2. Lowest energies of states with odd number of particles $N=1$ (squares) and $N=3$ (triangles). The values are given relative to the Fermi energy estimates in the text. The circles stand for the $N=3$ data after correction of the Fermi line shift.

estimated from the ground state energies at $N=2$ and $N=4$, and it becomes $\approx-24.6136 t$ for $U_{\text {eff }}=t$. The deviation from this value of the lowest-energy states for the odd-filled shell may signal the momenta at which a gap opens up for the quasiparticle excitations. Given the above mentioned shift of the Fermi line, though, one has to bear in mind that the Fermi level is not reached precisely at the points $\mathbf{P}=\left(\frac{3 \pi}{4}, \frac{\pi}{4}\right)$ and $\mathbf{P}=\left(\frac{\pi}{2}, \frac{\pi}{2}\right)$. This effect can be corrected by subtracting from the respective energies the shift from the Fermi level as measured for the $N=1$ quasiparticles. The final result can be seen in Fig. 2. The energy of the quasiparticles near $\mathbf{P}=$ $\left(\frac{3 \pi}{4}, \frac{\pi}{4}\right)$ and $\mathbf{P}=\left(\frac{\pi}{2}, \frac{\pi}{2}\right)$ turns out to be the same, within the precision of our computation, and slightly below the estimate from the mean value of the ground state energies for $N=2$ and $N=4$. On the contrary, the quasiparticle at $\mathbf{P}=(\pi, 0)$ has an energy well above the Fermi energy estimate. It is clear that a gap opens up for quasiparticle excitations, but only at the position of the saddle points. The value $\Delta$ of the gap for $U_{\text {eff }}=t, \Delta \approx 0.0017 t$, is sensibly greater than the energy difference between the lowest-energy states with $s$-wave and $d$-wave symmetry for $N=4$.

The conclusive evidence that the $d$-wave ground state at $N=4$ is built out of pairing of particles is given by the measure of pairing correlations with $d$-wave symmetry. We have computed the correlations of the operator

$$
O_{S C D}(\mathbf{q})=\sum_{\mathbf{k}}\left(\frac{g(\mathbf{k}+\mathbf{q})+g(\mathbf{k})}{2}\right) c_{\uparrow}^{+}(\mathbf{k}+\mathbf{q}) c_{\downarrow}^{+}(-\mathbf{k})
$$

with $g(\mathbf{p})=\cos \left(p_{x}\right)-\cos \left(p_{y}\right)$, in the ground state for $N=4$ at $U_{\text {eff }}=t$. The results are shown in Table II. The momenta that can be measured are enough to discern a high peak at $\mathbf{q}=0$ of the pairing correlations, together 
with a sharp decrease away from the origin. To establish some comparison, we may quote that the peak of the $s$ wave pairing correlations in the lowest-energy state of the $\mathrm{P}$ sector is $\approx 6.9$. Taking into account that the $d$-wave correlations decay by one order of magnitude across two lattice units in momentum space, the translation of the results to real space is that the binding of particles is correlated on the whole dimension of the cluster.

\begin{tabular}{|c|r|}
\hline \hline $\mathbf{q}$ & $\left\langle O_{S C D}(\mathbf{q}) O_{S C D}^{+}(-\mathbf{q})\right\rangle$ \\
\hline$(0,0)$ & 25.969 \\
$\left(\frac{\pi}{4}, 0\right)$ & 4.907 \\
$\left(\frac{\pi}{4}, \frac{\pi}{4}\right)$ & 8.526 \\
$\left(\frac{\pi}{2}, 0\right)$ & 3.262 \\
$\left(\frac{\pi}{2}, \frac{\pi}{4}\right)$ & 1.671 \\
$\left(\frac{\pi}{2}, \frac{\pi}{2}\right)$ & 1.059 \\
\hline \hline
\end{tabular}

TABLE II. Values of the pairing correlator for different momenta near the origin.

Although our results refer to a $8 \times 8$ cluster, they are robust enough to survive the extrapolation to larger lattices. The reason for that is the high degree of symmetry of the model before switching on the interaction with the Fermi sea. We have seen that, by focusing on the scattering processes at the Fermi level shell $\varepsilon=0$, the lowest-energy state with momentum $\mathbf{P}=(\pi, \pi)$ and those with $d$-wave and $s$-wave symmetry turn out to be degenerate. This is a universal feature of the interaction of states on the nested Fermi line, irrespective of lattice size. Our approach to the many-body problem starts by solving first the strong interaction in the highly degenerate Fermi level. While the interaction with the rest of excitations from the Fermi sea is relevant, as it resolves the mentioned degeneracy, its effect may be assessed within our weak-coupling renormalization group scheme.

The existence of the $d$-wave ground state with remarkable pairing correlations provides a microscopic basis for a long-standing conjecture. There has been much hope that a mechanism based on antiferromagnetic fluctuations may be the source of superconductivity in the copper-oxide materials. A different approach - though similar in essence - has put forward the idea that the Hubbard model should bear a form of Kohn-Luttinger superconductivity near half-filling. By stressing the relevance of the modes near the Fermi level shell at $\varepsilon=0$, our explicit construction of the $d$-wave ground state points at the processes with momentum transfer $\mathbf{Q}=(\pi, \pi)$ as the source of an attractive channel in the Hubbard model with bare repulsive interaction. Nesting of the saddle points at the Fermi level is at the origin of the relevance of such processes, which single out $d$-wave superconductivity as the dominant instability of the system.

I thank F. Guinea for useful comments on the manuscript. This work has been partially supported by the spanish Ministerio de Educación y Cultura Grant
PB96-0875.

[1] See, for instance, E. Dagotto, Rev. Mod. Phys. 66, 763 (1994).

[2] N. E. Bickers, D. J. Scalapino and S. R. White, Phys. Rev. Lett. 62, 961 (1989). P. Monthoux, A. V. Balatsky and D. Pines, Phys. Rev. B 46, 14803 (1992).

[3] J. González, F. Guinea and M. A. H. Vozmediano, Europhys. Lett. 34, 711 (1996); report cond-mat/9502095.

[4] D. Zanchi and H. J. Schulz, Phys. Rev. B 54, 9509 (1996). D. Z. Liu and K. Levin, Physica 275C, 81 (1997).

[5] E. Dagotto and J. Riera, Phys. Rev. Lett. 70, 682 (1993). D. Poilblanc, Phys. Rev. B 48, 3368 (1993). N. Bulut, D. J. Scalapino and S. R. White, Phys. Rev. B 47, 6157 (1993); Phys. Rev. B 50, 9623 (1994).

[6] Clear signatures of $d$-wave symmetry in ladder systems have been found by R. M. Noack, S. R. White and D. J. Scalapino, Phys. Rev. Lett. 73, 882 (1994); Europhys. Lett. 30, 163 (1995).

[7] A. I. Lichtenstein and M. I. Katsnelson, report cond-mat/9911320. Th. Maier et al., report condmat/0002352.

[8] T. Husslein et al., Phys. Rev. B 54, 16179 (1996). K. Kuroki and H. Aoki, Phys. Rev. B 56, R14287 (1997).

[9] M. Calandra and S. Sorella, report cond-mat/9911478.

[10] H. J. Schulz, Europhys. Lett. 4, 609 (1987). P. Lederer, G. Montambaux and D. Poilblanc, J. Phys. (Paris) 48, 1613 (1987). R. S. Markiewicz and B. G. Giessen, Physica 160C, 497 (1989). D. M. Newns et al., Phys. Rev. Lett. 69, 1264 (1992).

[11] J. V. Alvarez, J. González, F. Guinea and M. A. H. Vozmediano, J. Phys. Soc. Jpn. 67, 1868 (1998). C. J. Halboth and W. Metzner, Phys. Rev. B 61, 7364 (2000). C. Honerkamp et al., report cond-mat/9912358.

[12] J. González, F. Guinea and M. A. H. Vozmediano, report cond-mat/9905166, to be published in Phys. Rev. Lett.

[13] J. González, F. Guinea and M. A. H. Vozmediano, Nucl. Phys. B 485, 694 (1997).

[14] A. Parola et al., Phys. Rev. B 43, 6190 (1991), G. Fano, F. Ortolani and A. Parola, Phys. Rev. B 46, 1048 (1992).

[15] J. Von Neumann and E. P. Wigner, Physik. Z. 30, 467 (1929).

[16] Y. Asai and H. Katagiri, Phys. Rev. B 60, R13946 (1999), and private communication.

[17] J. González and J. V. Alvarez, Phys. Rev. B 56, 367 (1997).

[18] E. Dagotto, A. Nazarenko and A. Moreo, Phys. Rev. Lett. 74, 310 (1995). A. Moreo et al., Phys. Rev. B 51, 12045 (1995).

[19] This effect has been also remarked in D. Duffy and A. Moreo, Phys. Rev. B 52, 15607 (1995). See also M. Langer et al., Phys. Rev. Lett. 75, 4508 (1995).

[20] R. S. Markiewicz, J. Phys.: Condens. Matter 2, 665 (1990).

[21] A. Himeda and M. Ogata, report cond-mat/0003278. 